\title{
Attualità e prospettive dell'ecografia con mezzo di contrasto (CEUS) in nefrologia
}

\author{
Vincenzo Barbera ${ }^{1}$, Marco Federici ${ }^{2}$, Luca Di Lullo ${ }^{1}$, Giovanni Otranto ${ }^{1}$, Antonio Gorini ${ }^{1}$, Alberto Santoboni ${ }^{1}$ \\ ${ }^{1}$ UOC Nefrologia e Dialisi, Ospedale "L. Parodi Delfino», ASL ROMA G, Colleferro (RM) \\ ${ }^{2}$ UO Diagnostica per Immagini, Ospedale «L. Parodi Delfino», ASL ROMA G, Colleferro (RM)
}

\section{Current and future perspectives of contrast-enhanced ultrasonography (CEUS) in nephrology}

Contrast-enhanced ultrasonography (CEUS) is becoming an ultrasound device with many diagnostic applications. Employment of microbubbles as contrast media agents has allowed dynamic evaluation of micro- and macrovessels strengthening the characterization of ultrasonographic lesions not valuable with Power and Color Doppler analysis. CEUS does not involve ionizing radiations, then it's not harmful for kidney function. CEUS seems to be particularly helpful in distinguishing between benign cysts and malignancies according to Bosniak's classification. CEUS can also be applied to diagnose ischemic or traumatic issues, renal artery stenosis, autosomal dominant polycystic kidney disease and urinary infections or traumatic injuries and for kidney graft evaluation.

Further perspectives for CEUS lie in oncology for post-ablation kidney surveillance and in nanobubbles devices.

Keywords: CEUS, Microbubbles, Renal masses, TIC, Enhancement

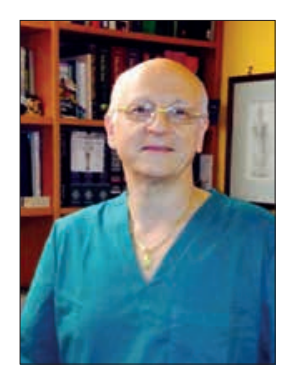

Introduzione

L'ecografia convenzionale in scala di grigi (US) rappresenta spesso la prima modalità di «imaging» utilizzata nello studio morfologico del rene. Essa consente di valutare le dimensioni renali, lo spessore e l'ecogenicità corticale rivelando altresi l'eventuale presenza di ostruzione, calcoli e/o masse. Tuttavia, qualora non si riesca a porre una corVincenza Barbera retta diagnosi, può rendersi necessario il ricorso ad altre tecnologie, come la tomografia computerizzata (CT) e/o la risonanza magnetica (MR) utilizzate per chiarire, confermare o confutare il sospetto diagnostico emerso dall'indagine US. A tal proposito, nonostante l'intuitiva importanza che la corretta diagnosi riveste, non si può non tenere conto della

Accepted: May 6, 2015

Published online: July 3, 2015

Indirizzo per la corrispondenza:

Dr. Vincenzo Barbera

UOC Nefrologia e Dialisi

Ospedale «L. Parodi Delfino»

Piazzale A. Moro 1

00034 Colleferro (RM)

vincenzo.barbera@aslromag.it relativamente elevata dose di radiazioni alle quali il paziente è sottoposto in corso di CT, variabile da circa 6 a $24 \mathrm{mSv}$ (1, 2) nel caso, per esempio, della regione addominale; inoltre, si stima che circa l'1.5-2\% di tutte le neoplasie sia attribuibile alle radiazioni utilizzate per tale procedura diagnostica (3). Nei pazienti con compromissione della funzione renale, la ripetizione dell'indagine come conseguenza di un followup diagnostico (p. es., in corso di neoplasie) deve essere attentamente considerata a causa del reiterato utilizzo del mezzo di contrasto ( $\mathrm{mdc}$ ) iodato. Per quanto riguarda invece la MR, vi sono segnalazioni che riguardano sia la tolleranza (p. es., claustrofobia) (4) che la sicurezza del paziente $(5,6)$, quest'ultima particolarmente per il rischio di fibrosi sistemica nefrogenica. Alla luce di tali considerazioni, in questi ultimi anni è andato sempre più affermandosi nella pratica clinica quotidiana l'utilizzo dell'ecografia con mezzo di contrasto (CEUS), che utilizza microbolle contenenti gas come "enhanced» e le sequenze armoniche tissutali per la visualizzazione della perfusione parenchimale. La valutazione delle curve renali intensità-tempo (TIC) aggiunge ulteriori opportunità per la valutazione del danno vascolare e del follow-up post-trapianto, in corso di trauma o nel monitoraggio successivo a procedure interventistiche, come l'ablazione con radiofrequenze. I vantaggi della CEUS sono ben documentati e includono la sua sicurezza (7-9), la semplicità di esecuzione, l'accettazione da parte del paziente e l'assenza di radiazioni ionizzanti. 


\section{Principi e metodologia}

\section{Mezzi di contrasto ecografici e principi fisici della CEUS}

Nel 1968, Gramiak e Shah (10) descrissero per la prima volta un "enhancement» ecografico dovuto a microbolle di aria prodotte da una soluzione salina introdotta nel cuore destro. Da allora i notevoli progressi ottenuti nel perfezionamento tecnologico delle apparecchiature, nell'imaging armonico e nelle tecniche ecografiche denominate contrasto-specifiche hanno consentito di ottimizzare la composizione dei mdc permettendo di giungere alle attuali sostanze di II generazione a base di microbolle contenenti gas inerti. L'efficacia di tali sostanze va prima di tutto ricercata nella loro composizione. Si tratta, infatti, di soluzioni liquide disperse di microgusci lipidici (microbolle), aventi un diametro medio compreso tra 2 e $5 \mathrm{~mm}$, che racchiudono un gas inerte avente bassa diffusività, così da aumentare la vita media delle microbolle stesse. Iniettate in circolo hanno lo scopo di incrementare, localmente e per un breve periodo, l'ecogenicità, consentendo, in tal modo, la visibilità nelle immagini ecografiche dei vasi sanguigni, compresi quelli di calibro molto ridotto non esplorabili con la metodica Doppler.

Le principali caratteristiche che un mdc ecografico deve possedere sono:

- facile diffusibilità nel comparto vascolare;

- elevata stabilità;

- capacità di modificare le proprietà acustiche dei tessuti;

- $\quad$ assenza di tossicità.

Le microbolle, per potersi disperdere nel sistema vascolare e attraversare il filtro polmonare, devono possedere un diametro inferiore a $10 \mathrm{~mm}$; le microbolle dei mdc di II generazione hanno un diametro compreso fra i 2 e i $5 \mathrm{~mm}$.

L'elevata stabilità in circolo è direttamente correlata al tempo di persistenza. Quest'ultimo, a sua volta, dipende da numerosi parametri legati alle caratteristiche geometriche delle microbolle, alle proprietà fisico-chimiche del gas in esse contenuto e alla membrana di rivestimento. Tali parametri sono riassunti in una formula derivata dalla legge di Laplace:

$$
T=\frac{r^{2} \cdot p}{2 D \cdot C s}
$$

in cui:

$\mathrm{T}=$ tempo di persistenza in circolo della microbolla;

$r=$ raggio della microbolla;

$\rho=$ densità del gas contenuto nella microbolla;

$\mathrm{D}=$ diffusibilità del gas attraverso la membrana

di rivestimento;

$\mathrm{C}_{\mathrm{s}}=$ costante di saturazione del gas nel sangue.

I mdc di I generazione erano composti di microbolle di

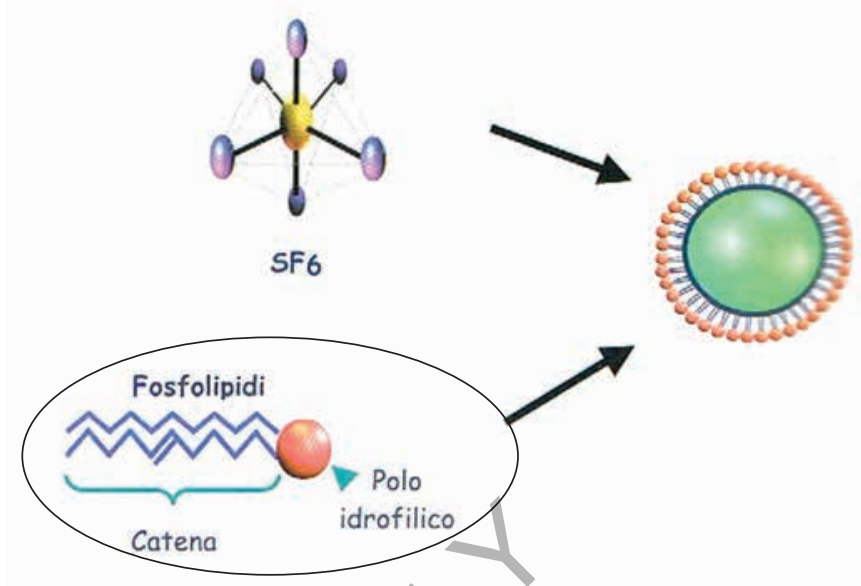

Fig. 1 - Microbolla di Sonovue $(2,5 \mu \mathrm{m})$ costituita da un guscio fosfolipidico (spessore $5 \mathrm{~nm}$ ) contenente esafluoruro di zolfo (SF6) gas idrofobico, stabile ed inerte.

aria stabilizzate da albumina, acido palmitico e galattosio; il loro rivestimento rigido non modificava il segnale e la pressione troppo elevata (indice meccanico 1.0-1.5) portava a una loro precoce rottura. Sono state quindi realizzate microbolle con membrane elastiche molto sottili contenenti gas inerti; grazie a una capsula molto soffice (possono essere paragonate a bolle di sapone), sono in grado di oscillare in risposta allo stimolo acustico generando onde riflesse; a pressioni anche molto ridotte (Indice Meccanico o $\mathrm{Ml}<0.10<100 \mathrm{KPa}$ ), non vengono distrutte e l'effetto contrastografico ha una maggiore durata. I mdc di II generazione (Fig. 1) attualmente in commercio sono microbolle contenenti esafluoruro di zolfo o perfluoropropano rivestite da una sottilissima membrana elastica costituita da fosfolipidi o da albumina. L'unico attualmente disponibile e commercializzato in Europa è il Sonovue (Bracco): sospensione acquosa di microbolle stabilizzate di esafluoruro di zolfo e membrana di fosfolipidi.

Una delle caratteristiche più importanti dei mdc ecografici di II generazione risiede nella loro capacità di interagire con gli ultrasuoni. Quando una microbolla nel sangue viene raggiunta da ultrasuoni direttamente provenienti da un trasduttore, inizia a oscillare inviando all'apparecchiatura ecografica frequenze fondamentali e frequenze multiple della fondamentale (armoniche): la microbolla stessa si trasforma in generatrice di ultrasuoni. Quando una bolla sferica di gas immersa in un liquido, come quella del mdc ecografico immersa nel torrente circolatorio, viene disturbata da una forza esterna (fascio ultrasonoro), modifica la sua forma generando tre fenomeni diversi:

a) riflessione degli ultrasuoni;

b) emissione di un segnale caratteristico senza rottura della bolla;

c) emissione di un segnale molto intenso a seguito della sua rottura. 


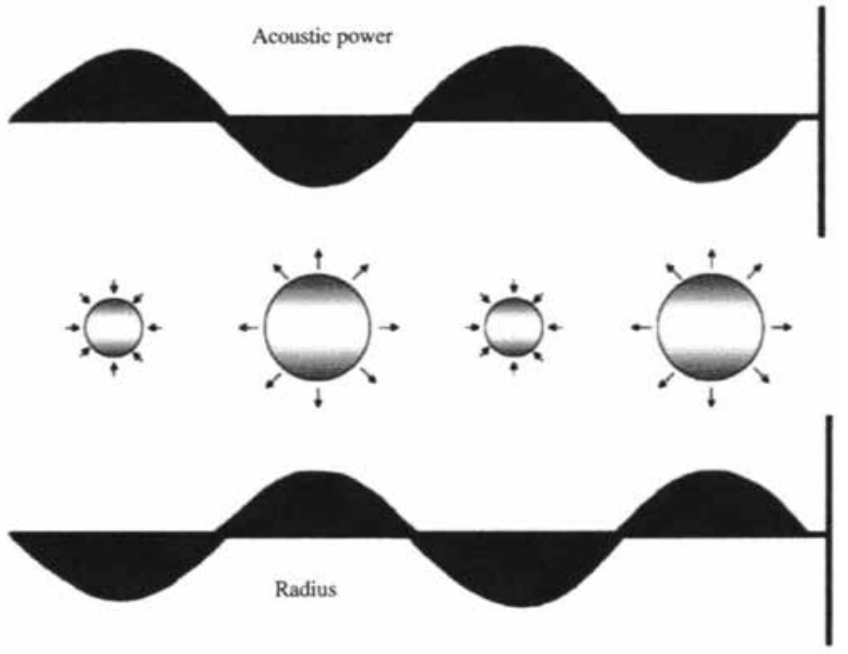

Fig. 2 - Comportamento lineare della microbolla che vibrandosi genera un ultrasuono caratteristico detto frequenza di risonanza.

Il verificarsi di questi tre fenomeni diversi dipende dal gas contenuto nella microbolla, dalla struttura elastica del suo rivestimento e dalle proprietà del fascio sonoro incidente. Per uno studio ecocontrastografico in real-time, è necessario sfruttare il secondo dei tre fenomeni elencati mantenendo il più a lungo possibile le microbolle integre; infatti, se la pressione acustica generata dal trasduttore (MI) non è così elevata da determinare la rottura della bolla, la stessa inizia a oscillare (vibrare), modificando il suo diametro attraverso una serie di contrazioni ed espansioni (Fig. 2). La bolla vibra con maggiore intensità a frequenze pari alla sua frequenza di risonanza che, per bolle aventi diametro inferiore a 7 micron, corrisponde alle frequenze comunemente utilizzate nella diagnostica ecografica (2$10 \mathrm{MHz}$ ). A $3.5 \mathrm{MHz}$, le microbolle oscillano a 3.5 milioni di contrazioni-espansioni/sec e generano un'intensità di echi di ritorno cento miliardi di volte maggiori rispetto a quelli delle cellule ematiche. Le frequenze generate dalle microbolle in vibrazione che originano echi di ritorno al trasduttore non contengono solo la frequenza di insonazione (frequenza fondamentale) ma anche frequenze multiple (doppie, triple, ecc.), definite, appunto, frequenze armoniche. Per tale motivo, gli apparecchi ecografici devono essere forniti di nuovi e specifici algoritmi che selezionano $i$ segnali provenienti soltanto dal mdc-US a sfavore delle frequenze degli echi generati dai tessuti. Tramite l'analisi di queste frequenze multiple verranno esaltate solo le strutture vascolari contenenti le microbolle del mdc-US.

Altra caratteristica fondamentale dei mdc ecografici è l'assenza di tossicità. In uno studio italiano eseguito su 28 centri (8), sono stati analizzati i dati relativi all'uso del Sonovue ${ }^{\circledR}$ relativamente agli eventi avversi (AE) su 23.188 ecografie addominali con mdc e la loro incidenza. Non è stato segnalato nessun $A E$ fatale; sono stati descritti $29 \mathrm{AE}$ di cui solo 2 rite- nuti seri (dispnea, broncospasmo, ipotensione e bradicardia con perdita di coscienza), 27 non seri (ipotensione moderata, cefalea, nausea e vomito) e 23 lievi (prurito, eritema). II tasso di incidenza degli AE seri è stato dello $0.0086 \%$. Nonostante la sicurezza, è tuttavia opportuno, in caso di CEUS, far firmare al paziente il consenso informato e avere a disposizione presidi sanitari per il primo intervento. Le principali controindicazioni all'impiego del mdc ecografico sono rappresentate da ipersensibilità nota nei riguardi dei componenti del mdc, sindrome coronarica acuta di recente insorgenza, cardiopatia ischemica clinicamente instabile, gravi aritmie, cardiopatia con shunt destro-sinistro, ipertensione polmonare, scompenso cardiaco severo (classe III-IV NYHA), uso di valvole cardiache, gravidanza e allattamento.

\section{Enhancement renale normale}

II circolo arterioso renale è rifornito da un singolo vaso tributario, rappresentato nei due lati dall'omonima arteria. Di conseguenza, il suo pattern di enhancement sarà evidentemente diverso da quello, per esempio, del fegato, provvisto di un duplice apporto ematico. A differenza dei mdc iodati e paramagnetici, le microbolle sono esclusivamente intravascolari e, non essendo eliminate attraverso il filtro renale, non determinano un effetto nefrografico o una fase escretoria. All'iniezione di un loro singolo bolo segue una precoce fase arteriosa con evidente differenziazione cortico-midollare (Fig. 3), della durata di circa 20-40"; segue una fase tardiva (45-120"), in cui si assiste alla scomparsa di tale differenziazione e al manifestarsi di un enhancement omogeneo corticale e midollare. Ricevendo circa il $25 \%$ della portata cardiaca, il rene si impregna rapidamente e intensamente rispetto ad altri visceri addominali; è possibile, perciò, valutare, in successione, anche il fegato (nei 3 minuti successivi) e la milza (che trattiene il contrasto fino al settimo minuto). Dal momento che il giudizio sulla vascolarizzazione di una lesione avviene per confronto diretto con il circostante parenchima, è evidente come il principale utilizzo clinico della CEUS consista nella valutazione e nella caratterizzazione delle lesioni focali.

\section{Curve intensità/tempo (TIC)}

Un'interessante applicazione clinica dei mdc ecografici consiste nella possibilità di ricavare informazioni quantitative riguardanti la perfusione tissutale. Tali metodiche sono riconducibili essenzialmente a due modalità, "passiva» e "attiva». Nell'approccio "passivo» l'utilizzo di basse energie di insonazione permette la registrazione del bolo di contrasto con minima distruzione di microbolle. Tuttavia, la tecnica più diffusa per quantificare i parametri perfusionali assoluti o quelli proporzionali al flusso ematico in una determinata area è quella di distruzione-riperfusione ("attiva»): quando tutte le microbolle in una determinata area vengono distrutte da impulsi di elevato $\mathrm{MI}$, il successivo riempimento dipende dalle 

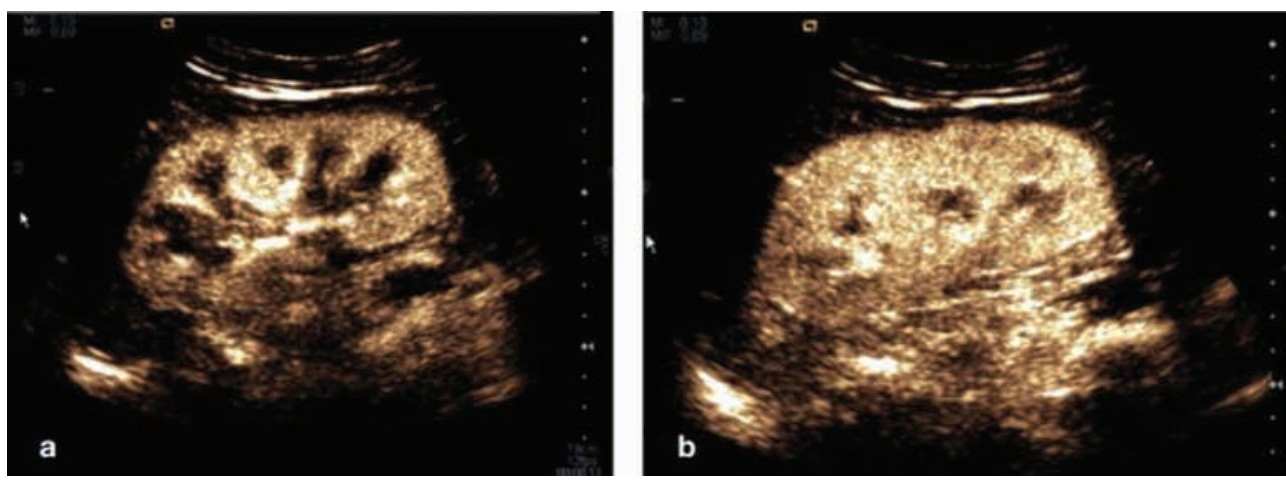

Fig. 3 - Normale enhancement renale. a) fase precoce (circa 15" dopo iniezione del $\mathrm{mdc}$ ): netta differenziazione della componente corticale e midollare; b) fase tardiva (60"): omogeneità del parenchimale renale.

nuove microbolle che entrano nella sezione dai tessuti adiacenti e può essere rilevato in maniera non distruttiva (basso MI). Tecnicamente, mantenendo la sonda ecografica in sede fissa per l'intero periodo, si invia una sequenza di impulsi ultrasonori predefiniti, inizialmente ad alta potenza e, in seguito, a intensità minore, finalizzati alla stimolazione armonica delle nuove microbolle in ingresso. Lo "slope» in ascesa della curva di riempimento ottenuta dipende dalla velocità media del flusso ematico nei vasi regionali. La percentuale di riempimento segue una curva il cui incremento iniziale dipende dalla velocità media di flusso della regione di interesse e il cui picco massimo indica la frazione di volume vascolare: il prodotto di queste grandezze è una misura proporzionale alla vera perfusione tessutale. I parametri perfusionali calcolati a partire dalla curva intensità/tempo sono numerosi: intensità del picco di segnale, tempo per il massimo enhancement («tempo di picco»), tempo per l'enhancement, area sotto la curva, gradiente positivo e durata dell'enhancement (Fig. 4).

\section{Lesioni focali del rene}

\section{Pseudomasse}

Con il termine «pseudomasse» si definisce un gruppo di varianti anatomiche renali che spesso possono simulare una lesione focale all'US. Tra queste, l'ipertrofia della colonna mesorenale (del Bertin), la persistenza delle lobature fetali e il "rene a dromedario» rappresentano quelle di più frequente riscontro e spesso avviate all'indagine CT con mdc (CE-CT) o MR per la risoluzione del dubbio diagnostico.

L'US è solitamente abbastanza accurata nel definire tali varianti e nell'escludere una massa renale. Un criterio fondamentale per la diagnosi di pseudomassa è il rilievo di un pattern ecostrutturale sovrapponibile a quello del parenchima circostante, sebbene talune differenze possano aversi in conseguenza della diversa ecoreflettività, riconducibile a difformità nell'orientamento tissutale. Alla CEUS, l'area di interesse mostra lo stesso pattern di enhancement del parenchima normale. È stata dimostrata una completa concordanza tra CEUS e CE-CT o MR nella caratterizzazione delle pseudomasse renali non univocamente diagnosticate con il solo utilizzo dell'US e del color Doppler (11). Una volta che tali reperti siano stati così confermati, non è più necessario il ricorso ad alcuna procedura diagnostica, con evidenti vantaggi per la serenità del paziente.

\section{Infezioni}

In accordo con le Linee Guida dell'Associazione Urologi Europei, la diagnosi di pielonefrite acuta non complicata è basata sul quadro clinico e sui reperti bioumorali. L'utilità dell'US, in tali casi, consiste nel valutare la morfometria renale e la presenza o meno di cicatrici corticali (pregressi episodi pielonefritici), nel documentarne una sottostante alterazione strutturale, ovvero l'eziologia, e nel confermare o meno l'esistenza di un'ostruzione (calcolo) o di un ascesso. Ulteriori indagini dovrebbero essere considerate qualora il paziente sia ancora febbrile dopo 72 ore di trattamento. In questi casi, sebbene il rene possa apparire normale all'esame convenzionale, aree di focale riduzione dell'enhancement, soprattutto di aspetto cuneiforme, possono essere evidenziate alla CT in corso di pielonefrite acuta batterica (12). Allo stesso modo, aree di mancato enhancement, interpretabili come zone di infezione focale, possono essere evidenziate dalla CEUS. Quest'ultima, rispetto alla CE-CT, ha mostrato di possedere un'elevata sensibilità e un valore predittivo positivo del $100 \%$ nella diagnosi di pielonefrite acuta (13). Valutazioni ripetute nel tempo possono essere di aiuto nel dimostrare la mancata risoluzione del processo e la precoce formazione di un ascesso in quei pazienti che non rispondono o rispondono solo parzialmente a un'idonea terapia antibiotica. In caso di ascessualizzazione, può evidenziarsi un'area parenchimale disomogenea priva di enhancement, circondata da un intenso uptake periferico. Pertanto, la CEUS dovrebbe essere riservata a tali specifici casi piuttosto che essere utilizzata in maniera estensiva.

\section{Lesioni cistiche renali}

Le lesioni cistiche renali sono un reperto molto comune e la maggioranza di esse ha caratteri di non evolutività. La loro incidenza aumenta in funzione dell'età e delle diverse casistiche, potendo variare dal $23.3 \%$ tra i $50-59$ anni fino a circa il $32.6 \%$ 

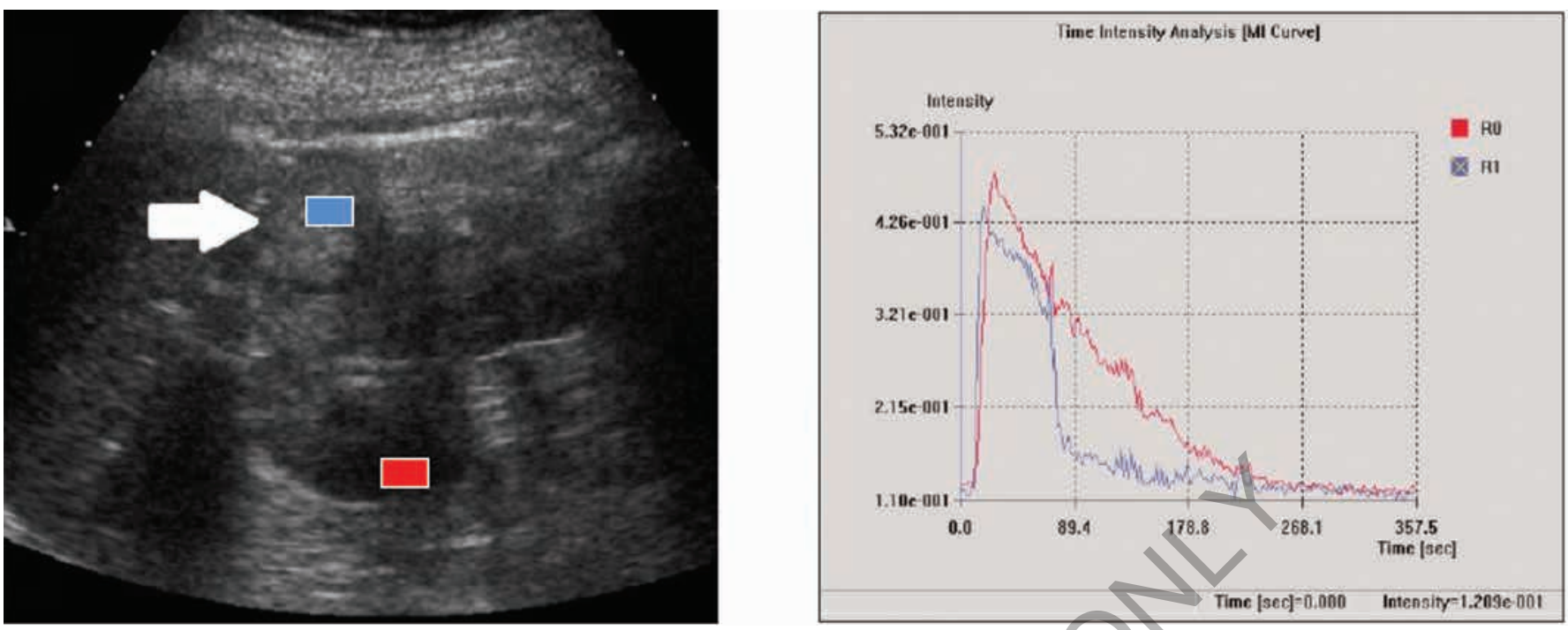

Fig. 4 - TIC ottenuta da adenocarcinoma renale a cellule chiare. Nel tessuto neoformato (color bluette) rispetto al parenchima renale sano (colore rosso) si nota una curva caratterizzata da un precoce tempo di arrivo delle microbolle con rapido calo dell'intensità di segnale, precoce ritorno al «baseline» e più rapidi wash-in e wash-out dell'AUC.

oltre i 60 anni (14). Talora, può risultare difficoltoso distinguere le cisti benigne dai tumori cistici, anche in considerazione del fatto che circa il $10 \%$ dei tumori renali si presenta come una massa cistica. Per molto tempo, lo studio di tali neoformazioni è stato realizzato mediante indagine CT e, a tale proposito, nel 1986, Bosniak (15) propose una classificazione delle cisti renali, successivamente rivisitata (Tab. I), basata sull'aspetto tomodensitometrico ( $\mathrm{ma}$, per certi versi, estensibile anche all'indagine US e MR), che suddivideva le cisti in quattro classi in funzione del crescente rischio neoplastico con conseguente diverso approccio terapeutico.

L'US è, generalmente, superiore alla CT nella dimostrazione dell'architettura interna delle lesioni cistiche in termini di contenuto fluido, di presenza o meno di sepimentazioni e sottili nodulazioni e di alterazioni parietali. Le cisti renali semplici vengono studiate assai meglio con l'impiego dell'imaging armonico che consente di eliminare gli echi interni di basso livello, riducendo, in tal modo, il rumore di fondo. Tuttavia, qualunque neoformazione cistica che all'US non corrisponda ai rigidi criteri di benignità (Tab. I) è, per definizione, complicata e richiede ulteriori approfondimenti diagnostici. Questi consistono nel valutare la presenza o meno, dopo la somministrazione del mdc, di un enhancement da parte della componente solida che, ovviamente, non può essere evidenziata in B-mode. Analogamente alla CE-CT, la CEUS consente di dimostrare molti dei caratteri di malignità come ispessimenti della parete delle cisti e presenza di setti e nodularità, mentre è meno sensibile nel rilevare la presenza di lesioni calcifiche. In maniera simile alla CE-CT, ogni significativo uptake di mdc internamente a una lesione cistica, eccezion fatta per i setti, dovrebbe essere considerato come un potenziale indice di malignità. Numerosi studi hanno dimostrato un'ottima concordanza tra CEUS e CE-
CT nella classificazione delle cisti complesse utilizzando il sistema di Bosniak, in special modo nel differenziare le forme che necessitano dell'intervento del chirurgo da quelle non chirurgiche (16-18). Occasionalmente, data la composita architettura di tali lesioni, alcune di esse restano indeterminate anche con la CT con valori di enhancement di soli 10-20 unità Hounsfield (HU): in tali condizioni, si può individuare un ruolo potenziale della CEUS in associazione con la CT. È il caso delle lesioni tipo Bosniak IIF, in cui la CEUS sta assumendo un ruolo crescente influenzando il percorso decisionale in senso chirurgico od osservazionale. Tuttavia, considerando l'esperienza clinica di 30 anni della classificazione CT di Bosniak e l'utilizzo clinico relativamente recente della CEUS, è opportuno che tutte queste lesioni continuino a essere valutate anche con la $\mathrm{CT}$, poiché questa potrebbe rendersi necessaria per il loro staging qualora esse risultino potenzialmente maligne. Nel caso di condizioni a elevato rischio neoplastico, quale potrebbe essere la malattia di von Hippel-Lindau, tali difficoltà diagnostiche sono amplificate come conseguenza delle molteplici piccole cisti che possono andare incontro a un processo maligno. L'innocuità della CEUS rende tale indagine di prima scelta in questi pazienti, soprattutto giovani, che hanno necessità di monitorare l'evolutività di tali lesioni. Non vi è, però, ancora una prova definitiva della sua efficacia, poiché molti di questi tumori sono assai piccoli.

\section{Tumori renali}

\section{Tumori renali benigni}

Circa il $60-85 \%$ dei tumori renali benigni (BRT) ha dimensioni inferiori ai $4 \mathrm{~cm}$ (19-22). La maggioranza di questi è rappresentata da oncocitomi (circa 74\%) e da angiomiolipomi (14\%), 
TABELLA I - Classificazione secondo bosniak delle cisti renali

\begin{tabular}{|c|c|}
\hline Categoria & Descrizione delle caratteristiche \\
\hline | & $\begin{array}{l}\text { Cisti benigna semplice con parete sottile simile ad un capello non contenente sepimenti calcificazioni o componenti solide; } \\
\text { possiede la densità dell'acqua e non presenta alcun enhancement. Non richiede alcun intervento. }\end{array}$ \\
\hline ॥ & $\begin{array}{l}\text { Cisti benigna con pochi sepimenti sottili come capelli. Minute calcificazioni possono essere presenti nella parete o nei } \\
\text { sepimenti. Lesioni }<3 \mathrm{~cm} \text { uniformemente iperdense, con pareti ben definite e che non presentato enhancement. Non } \\
\text { richiede alcun intervento. }\end{array}$ \\
\hline IIF & $\begin{array}{l}\text { Le cisti contengono più sepimenti sottili come capelli. Può osservarsi (non misurabile) minimo enhancement di un sepi- } \\
\text { mento o della parete; può esservi minimo ispessimento dei setti o della }\end{array}$ \\
\hline & $\begin{array}{l}\text { parete. Le cisti possono contenere calcificazioni nodulari e spesse, ma non vi è alcuna componente solida che presenti } \\
\text { enhancement. Sono incluse in questa categoria lesioni totalmente intrarenali } \geq 3 \mathrm{~cm} \text { ad alta densità che non presentano } \\
\text { alcun enhancement. Tali lesioni sono generalmente a margini ben definiti. Si ritiene che siano benigne ma richiedono } \\
\text { follow-up per dimostrare tale caratteristica (stabilità nel tempo). }\end{array}$ \\
\hline III & $\begin{array}{l}\text { Lesioni cistiche con pareti lisce o irregolarmente ispessite o con setti in cui è presente un enhancement misurabile; queste } \\
\text { masse necessitano generalmente d'intervento chirurgico, poiché non è possibile escludere una neoplasia. Comprendono } \\
\text { le cisti complicate (cistiche o emorragiche), il nefroma cistico multiloculare. Necessitano di diagnosi istologica. }\end{array}$ \\
\hline IV & $\begin{array}{l}\text { Masse cistiche maligne che possiedono tutte le caratteristiche della classe III ma contenenti una componente di tessuto } \\
\text { con enhancement distinto da quello delle pareti o dei setti. Sono masse chiaramente maligne e debbono essere rimosse. }\end{array}$ \\
\hline
\end{tabular}

mentre, nel 4\% di casi, si tratta di adenomi papillari $(19,21)$.

Gli oncocitomi rappresentano circa il $5 \%$ delle masse renali solide $(23,24)$. Vengono generalmente considerati lesioni benigne, sebbene talora risultino difficilmente differenziabili, anche istologicamente, dal carcinoma a cellule renali (RCC) cromofobo (25). La diagnostica per immagini di tali neoformazioni è assai complessa per la mancanza di caratteri distintivi. In uno studio CE-CT condotto da Choudhary et al (26) su 21 pazienti con oncocitoma, solo il 18\% delle lesioni presentava una cicatrice fibrosa centrale, spesso descritta come caratteristica dell'oncocitoma. Tale reperto portò gli Autori a concludere che la CE-CT non era in grado di fornire immagini tipiche in presenza di un oncocitoma (26). Attualmente, vi sono solo pochi report riguardanti l'impiego della CEUS nella diagnosi di oncocitoma. Strobel et al in nessuno di tre pazienti con oncocitoma riuscirono a evidenziare la presenza di una cicatrice; inoltre, nella fase precoce, i 2/3 di essi risultarono ipervascolari e il restante terzo risultò ipovascolare, mentre, nelle fasi tardive, tutti mostrarono un comportamento ipovascolare rispetto al parenchima renale (27). Tamai et al descrissero come caratteristica dell'oncocitoma una configurazione dei vasi neoformati "a ruota raggiata» (28). Wu et al., esaminando cinque casi di oncocitoma con la CEUS, trovarono, in tre di essi, aree centrali irregolari di non-enhancement, che correlarono alla presenza della cicatrice fibrosa; per contro, essi non riuscirono a dimostrare, in nessuno dei cinque casi, il pattern vascolare a ruota raggiata. Gli Autori conclusero che la distinzione dell'oncocitoma dal RCC mediante tecniche radiologiche risulta assai difficoltoso e presenta scarso valore predittivo (29). Possiamo, pertanto, affermare che tali lesioni non possono essere differenziate utilizzando la CEUS $(28,30,31)$.

L'angiomiolipoma (AML) è un tumore mesenchimale formato da proporzioni variabili di tessuto adiposo, cellule muscolari lisce di aspetto fusiforme-epitelioide ed esili vasi sanguigni $(23,24)$. Per la sua componente adiposa, risulta facilmente identificabile alla CT nella fase di non-enhancement; tuttavia, fino al 54\% di AML istologicamente accertati può non essere radiologicamente differenziato da un RCC per la ridotta percentuale di componente adiposa (27). Dal momento che il principale obiettivo della diagnostica per immagini delle masse renali consiste nel differenziare quelle benigne dai RCC, la CEUS si candida a rivestire un ruolo di primo piano. In uno studio retrospettivo condotto da Xu et al., tale metodica ha dimostrato un'eccellente capacità nel distinguere il RCC dall'AML a scarsa componente adiposa (LFAML) (32). Il comportamento del LFAML all'ecografia con contrasto si caratterizza per un enhancement omogeneo che persiste a lungo durante le fasi cortico-midollare e tardiva; viceversa, il RCC presenta un rapido washout e un enhancement disomogeneo con rinforzo della rima perilesionale. Recentemente, uno studio retrospettivo ha confermato i reperti descritti (33). Furono arruolati pazienti con sospetto RCC e, prima della rimozione chirurgica, sottoposti a CEUS e a ecografia convenzionale. Il comportamento distintivo tra i due tipi di tumori consisteva in un lento enhancement centripeto nella fase corticale (83.3\% LFAML vs $1.9 \% \mathrm{RCC}$ ) con una fase di picco omogenea (100\% LFAML vs $34.3 \%$ RCC). L'associazione di questi due criteri CEUS rappresentava il principale elemento distintivo tra i due tipi di masse renali; il valore predittivo positivo era del $100 \%$ per il LFAML e del $\mathbf{9 7 . 2 \%}$ per il RCC. Viceversa, non fu possibile rendere evidenti differenze di ecogenicità né di segnale eco-color Doppler. Questi risultati sembrano suggerire importanti prospettive per la CEUS nella diagnostica differenziale tra LFAML e RCC, sebbene il gold standard test, vale a dire la CE-CT, non sia stato al momento utilizzato per valutare se la CEUS abbia o 
meno degli elementi di superiorità o almeno capacità simili e se esista una possibile correlazione tra dimensioni del tumore e pattern di enhancement.

\section{Tumori renali maligni}

La neoplasia renale di più frequente riscontro è rappresentata dal carcinoma a cellule renali (RCC), responsabile di circa il 90\% di tutti i tumori maligni. Istologicamente, la variante più comune è quella a cellule chiare che si rinviene nel $70 \%$ di tutti i RCC (34). Le dimensioni della neoplasia sono generalmente correlate alla sua malignità, sebbene tale caratteristica possa ritrovarsi anche nelle masse renali più piccole (19-22). Allo stesso modo, il tasso di crescita, il grado di malignità e lo stadio del tumore aumentano con l'accrescersi delle dimensioni $(19,35)$. A tale proposito Jiang et al $(36)$ e Xu et al (37) analizzarono retrospettivamente, con l'impiego della CEUS, le caratteristiche del RCC in relazione con le sue dimensioni. $\mathrm{Fu}$ osservato un enhancement disomogeneo in tumori $>3 \mathrm{~cm}$ (87-91\%) rispetto al $28-55 \%$ di quelli $\leq 3 \mathrm{~cm}$. Dal momento che, come avemmo modo di dire in precedenza, I'AML mostra un enhancement omogeneo, ciò comporterebbe che la CEUS è in grado di differenziare le masse maligne da quelle benigne solo per dimensioni $<3 \mathrm{~cm}$. Inoltre, è possibile che il pattern di enhancement rappresenti solo uno dei molteplici aspetti utili per tale determinazione, conclusione, quest'ultima, alla quale giunse anche lo studio di Xu et al (37). La combinazione di iper-o isoenhancement, di enhancement perilesionale e di washout nella fase tardiva sembra, invece, essere suggestiva di RCC. Lo studio pilota di Gerst et al (38), condotto su 34 pazienti (di cui 23 con documentato carcinoma renale a cellule chiare), confermò che la modalità di washout e il grado di enhancement, la misura quantitativa dell'intensità di picco e gli aspetti di disomogeneità ecostrutturale in scala di grigi potevano essere utili nel differenziare i carcinomi renali a cellule chiare dagli altri istotipi. Infine, Ignee et al (39) dimostrarono che la CEUS era sovrapponibile alla CE-CT nello "staging» del RCC, in quanto risultò in grado di stadiare correttamente l' $88 \%$ dei pazienti vs il $91 \%$ della CE-CT. Al contrario, Tamai et al (28) consigliano il ricorso alla CEUS solo nel caso in cui la CE-CT non riesca a dimostrare un enhancement della massa e, pertanto, nei tumori ipovascolari. Per i tumori ipervascolarizzati, la sensibilità e la specificità per la CEUS e la CE-CT erano, rispettivamente, del $94.4 \%$ e del $45.5 \%$ vs $88.95 \%$ e $72.2 \%$. Tali positivi dati preliminari sul valore diagnostico della CEUS necessitano, tuttavia, di ulteriori conferme ottenibili da studi prospettici multicentrici.

\section{Lesioni traumatiche}

La CT, meglio se multidetettore multifase, rappresenta il gold standard per la valutazione del paziente traumatizzato. In virtù della sua elevata sensibilità e panoramicità, tale tecnica consente di valutare sia l'aspetto parenchimale sia quello va- scolare ed escretore nel politrauma o nel trauma renale. Tuttavia, l'avvento dei mdc ecografici e il loro impiego hanno fornito nuove opportunità nella valutazione clinica dei traumi addominali. Catalano et al (40) hanno dimostrato come la CEUS sia una metodica promettente nella diagnostica dei traumi splenici, essendo più accurata e dettagliata rispetto all'US ed essendo correlata meglio con la CT. L'ecocontrastografia migliora la sensibilità dell'US dal $45.7 \%$ al $91.4 \%$ (41); essa permette una migliore definizione dei limiti della lesione e del parenchima normale nonché la sua estensione alla capsula, che rappresenta un importante criterio per il ricorso al chirurgo.

Nel caso del rene, la CEUS potrebbe essere utile nei traumi minori con ematuria o nei pazienti sottoposti a procedure potenzialmente lesive nelle 12 ore precedenti (p. es., litotripsia extracorporea). Ciò consentirebbe uno screening migliore dei soggetti con lesione renale ma anche di eventuali altri organi coinvolti (42). Lecografia con mdc di II generazione può essere, altresì, considerata come in grado di avviare alla valutazione CT quei pazienti sicuramente positivi, per documentare l'integrità della via escretrice e del peduncolo vascolare. Tale metodica non può essere applicata al soggetto politraumatizzato stabile che deve essere studiato con esame CT comprendente distretti corporei multipli.

\section{Trapianto renale}

Grazie al suo posizionamento relativamente superficiale e alle limitate escursioni respiratorie, il rene trapiantato ben si presta a essere valutato con mdc ecografico. Vi è, infatti, un ampio range di potenziali insulti in grado di determinare una disfunzione del graft sia acutamente, nell'immediato periodo post-operatorio, sia più tardivamente in maniera cronica. Subito dopo il trapianto, una volta escluse la condizione di idronefrosi e la presenza di eventuali infezioni, le cause più frequenti di ritardata ripresa funzionale sono rappresentate dalla necrosi tubulare acuta (ATN) e dal rigetto acuto (AR). Tali condizioni sono clinicamente subdole, in quanto si manifestano con segni estremamente aspecifici e, talora, in maniera addirittura silente. L'esame eco-color Doppler con valutazione della curva velocitometrica (calcolo degli indici di resistenza, RI), eseguito in maniera seriata, consente un certo giudizio sulla funzionalità del trapianto. L'evidenza di valori di RI persistentemente/ progressivamente elevati pone indicazione alla biopsia per una diagnosi definitiva di certezza. Gli studi tesi a investigare l'impiego dei mdc ecografici nelle complicanze precoci hanno evidenziato, nei pazienti con AR, una ritardata perfusione parenchimale nei confronti di quelli con tempestivo funzionamento del graft (43) e rispetto a coloro i quali presentino un'ATN istologicamente accertata (44). Anche se assai promettenti, tali risultati sono ancora «in fieri», poiché la biopsia renale rimane comunque il "gold standard» per la diagnosi.

La CEUS è stata testata anche nel monitoraggio della terapia immunosoppressiva (45). Alcuni Autori hanno persino dimostrato l'esistenza di una correlazione tra la perfusione del 
graft ottenuta con metodiche quantitative e il $\operatorname{GFR}(46,47)$ nonché tra $\mathrm{TIC}_{\mathrm{s}}$ e nefropatia cronica da trapianto (48).

La diagnosi di steno-occlusione dell'arteria e/o della vena renale è solitamente effettuata con esame eco-color Doppler. Tuttavia, in tali situazioni, la CEUS può fornire talune evidenze che possono risultare particolarmente utili qualora il paziente presenti indicazioni all'espianto del graft. In caso di occlusione arteriosa suscettibile di riparazione chirurgica, la CEUS riveste un ruolo importante nel follow-up della perfusione post-intervento, altrimenti difficile da valutare con l'imaging convenzionale. Altra importante indicazione è rappresentata dal paziente con ridotta o assente perfusione di una regione polare del rene. Talora, risulta impossibile riuscire a stabilire con l'US se tale aspetto sia reale o artefattuale, mentre l'ecocontrastografia ne permette una corretta differenziazione entro 30" dall'iniezione delle microbolle.

\section{Limiti della CEUS}

Nonostante i promettenti risultati conseguiti dalla CEUS nella differenziazione delle masse renali, è opportuno considerare alcune sue limitazioni che ne consentano un utilizzo ancora più vantaggioso. I dati quantitativi ottenibili con la metodica TIC sono notevolmente influenzati dalla localizzazione della lesione (superficiale vs profonda), soprattutto nei pazienti obesi, e dalla presenza di meteorismo intestinale, condizioni che riducono notevolmente la qualità delle immagini. Inoltre, l'ecocontrastografia, non permettendo la simultanea visualizzazione comparativa dei due reni, impedisce di cogliere le differenze di intensità e tempistica dell'enhancement parenchimale, cosa sempre possibile con la CT e la MR. Dal momento che le microbolle che compongono il contrasto, a causa delle loro dimensioni, non filtrano attraverso i glomeruli, non è possibile ottenere la fase pielografica. Di conseguenza, mentre si potrà sempre eseguire un'URO-CT, non sarà mai possibile un esame URO-US.

Essendo la metodica operatore-dipendente e dato che non esistono ancora indicazioni univoche per l'interpretazione delle immagini, sono richiesti esecutori con una buona esperienza nel campo, al fine di minimizzare la variabilità interindividuale. Infine, la FDA non ha ancora autorizzato l'impiego delle microbolle nella diagnostica radiologica delle masse renali (49).

\section{Prospettive della CEUS}

L'aumentato ricorso clinico alle tecniche di ablazione renale ha aperto interessanti prospettive per la CEUS. Infatti, tali tecniche, sia quelle che utilizzano le radiofrequenze (RFA) che quelle che utilizzano la crioablazione (CA), si sono rivelate in grado di preservare la funzione renale residua e, dal punto di vista prognostico, efficaci nel medio termine (50). Tuttavia, il tasso di recidive e di tumori residui è ancora elevato rispetto alla nefrectomia parziale e, pertanto, in tali casi, è necessario un follow-up radiologico più intensivo per diagnosticare precocemente eventuali ricomparse.
Un interessante campo di ricerca riguarda l'utilizzo delle microbolle per applicazioni cliniche diverse da quelle strettamente diagnostiche. Essendo trasportate dalla corrente ematica, tali microparticelle potrebbero essere impiegate per veicolare sostanze terapeutiche precedentemente inserite al loro interno. In tal modo, esse potrebbero raggiungere organi e tessuti altrimenti difficilmente accessibili per un rilascio controllato ed esclusivamente locale del farmaco. Una tale metodica avrebbe innegabili vantaggi in tutte quelle terapie, in primis oncologiche, in cui gli effetti collaterali delle sostanze utilizzate non sono trascurabili. L'efficacia della penetrazione all'interno delle cellule dei principi così rilasciati potrebbe essere aumentata impiegando soluzioni disperse di nanobolle, formate, cioè, da gusci lipidici del diametro di poche centinaia di nanometri (436.8 $\pm 5.7 \mathrm{~nm}$ ). È noto, infatti, che le nanobolle per valori sufficientemente elevati dell'intensità dell'onda acustica incidente possono diventare nuclei di cavitazione: se questo fenomeno si realizza in prossimità delle membrane cellulari, esso provoca, in queste ultime, l'apertura di nanopori del diametro di circa $300-700 \mathrm{~nm}$, aventi una vita media di 20-50 millisecondi. Questo fenomeno, che va sotto il nome di sonoporazione, aumenta la permeabilità cellulare favorendo la possibilità di ingresso all'interno delle cellule di sostanze farmacologicamente attive.

\section{Conclusioni}

L'ecografia con mezzo di contrasto (CEUS), anche se inizialmente utilizzata per la valutazione delle lesioni epatiche, ha guadagnato un posto di prestigio nello studio della patologia renale. In tale ambito, la CEUS rappresenta una metodica sicura, ben tollerata e riproducibile, consentendo di evitare, in taluni casi, il ricorso alla CT o alla MR. Un suo evidente vantaggio è rappresentato dalla mancanza di nefrotossicità. Le indicazioni nefrologiche per le quali è stata più ampiamente impiegata sono le valutazioni delle pseudomasse, delle masse renali e della patologia cistica e traumatica, nelle pielonefriti e nel follow-up del rene trapiantato. Risultati incoraggianti sembrano ottenersi nel monitoraggio post-trattamenti angiogenetici e nelle tecniche interventistiche di crioablazione e di ablazione con radiofrequenze dei tumori renali.

\section{Disclosures}

Financial support: No financial support was received for this submission. Conflict of interest: The authors have no conflict of interest.

\section{Bibliografia}

1. Fujii K, Aoyama T, Koyama $\mathrm{S}$, et al. Comparative evaluation of organ and effective doses for paediatric patients with those for adults in chest and abdominal CT examinations. $\mathrm{Br} J$ Radiol. 2007;80:657-67.

2. Van Unnik JG, Broerse JJ, Geleijns J, et al. Survey of CT techniques and absorbed dose in various Dutch hospitals. $\mathrm{Br} \mathrm{j}$ Radiol. 1997; 70:367-71. 
3. Brenner DJ, Hall EJ. Computed tomography-an increasing source of radiation exposure. N Engl J Med. 2007;357:2277-84.

4. Eshed I, Althoff CE, Hamm B, et al. Claustrophobia and premature termination of magnetic resonance imagin examinations. J Magn Reson Imaging. 2007;26:401-4.

5. Shellock FG, Spinazzi A. MRI safety update 2008: part 1, MRI contrast agents and nephrogenic systemic fibrosis. AJR Am J Roentgenol. 2008;191:1129-39.

6. Shellock FG, Spinazzi A. MRI safety update 2008: part 2, screening patients for MRI. AJR Am J Roentgenol. 2008;191:1140-9.

7. Jacobsen JA, Oyen R, Thomsen HS, et al. Safety of ultrasound contrast agents. Eur Radiol. 2005;15:941-5.

8. Piscaglia F, Bolondi L. The safety of SonoVue in abdominal applications:retrospective analysis of 23188 investigations. UItrasound Med Biol. 2006;32:1369-75.

9. Torzilli G. Adverse effects associated with SonoVue use. Expert Opin Drug Saf. 2005;4:399-401.

10. Gramiak R, Shah PM. Echocardiography of the aortic root. Invest Radiol. 1968;3(5):356-66.

11. Mazziotti S, Zimbaro F, Pandolfo A, et al. Usefulness of contrast-enhanced ultrasonography in the diagnosis of renal pseudotumors. Abdom Imaging. 2010;35:241-5.

12. Craig WD, Wagner BJ, Travis MD. Pyelonephritis: radiologicpathologic review. RadioGraphics. 2008;28:327-8. 255-77;quiz.

13. Mitterberger $M$, Pinggera GM, Colleselli $D$, et al. Acute pyelonephritis: comparison of diagnosis with computed tomography and contrast-enhanced ultrasonography. BJU Int. 2008;101:341-4.

14. Marumo K, Horiguchi $Y$, Nakagawa $K$, et al. Incidence and growth pattern of simple cysts of the kidney in patients with asymptomatic microscopic hematuria. Int J Urol. 2003;10:63-7.

15. Bosniak MA. The current radiologic approach to renal cysts. Radiology. 1986;158:1-10.

16. Ascenti G, Mazziotti S, Zimbaro G, et al. Complex cystic renal masses: characterization with contrast-enhanced US. Radiology. 2007;243:158-65.

17. Clevert DA, Minaifar N, Weckbach S, et al. Multislice computed tomography versus contrast-enhanced ultrasound in evaluation of complex cystic renal masses using the Bosniak classification system. Clin Hemorheol Microcirc. 2008;191:1239-49.

18. Park BK, Kim B, Kim SH, et al. Assessment of cystic renal masses based on Bosniak classification: comparison of CT and contrastenhanced US. Eur J Radiol. 2007;61:310-4.

19. Frank I, Blute ML, Cheville JC, et al. Solid renal tumors: an analysis of pathological features related to tumor size. J Urol. 2003;170:2217-20.

20. Murphy AM, Buck AM, Benson MC, et al. Increasing detection rate of benign renal tumors: evaluation of factors predicting for benign tumor histologic features during past two decades. Urology. 2009;73:1293-7.

21. Thompson RH, Kurta JM, Kaag M, et al. Tumor size is associated with malignant potential in renal cell carcinoma cases. J Urol. 2009;181:2033-6.

22. Tsivian M, Mouraviev V, Albala DM, et al. Clinical predictors of renal mass pathological features. BJU Int. 2011;107:735-40.

23. Lopez-Beltran A, Scarpelli M, Montironi R, et al. 2004 WHO classification of the renal tumors in adults. Eur Urol. 2006;49:798-805.

24. Lopez-Beltran A, Carrasco JC, Cheng L, et al. 2009 update on the classification of renal epithelial tumors in adults. Int J Urol. 2009;16:432-43.

25. Breda A, Treat EG, Haft-Candell L, et al. Comparison of accuracy of 14-,18- and 20-G needles in ex-vivo renal mass biopsy: a prospective blinded study. BJU. 2010;105:940-5.

26. Choudhary S, Rajesh A, Mayer NJ, et al. Renal oncocytoma: CT features cannot reliably distinguish oncocytoma from other renal neoplasms. Clin Radiol. 2009;64:517-22.

27. Haendl T, Strobel D, Legal W, et al. Renal cell cancer does not show a typical perfusion pattern in contrast-enhanced ultrasound. Ultraschall Med. 2009;30:58-63.

28. Tamai H, Takiguchi Y, Oka M, et al. Contrast-enhanced ultrasonography in the diagnosis of solid renal tumors. J Ultrasound Med. 2005;24:1635-40.
29. Wu Y, Du L, Li F, et al. Renal oncocytoma: contrast-enhanced sonographic features. J Ultrasoud Med. 2013;32:441-8.

30. Fan $L$, Lianfang $D$, Jinfang $X$, et al. Diagnostic efficacy of contrast-enhanced ultrasonography in solid renal parenchyma lesions with maximum diameters of $5 \mathrm{~cm}$. J Ultrasound Med. 2008;27:875-85.

31. Lane BR, Aydin H, Danforth TL, et al. Clinical correlates of renal angiomyolipoma subtypes in 209 patients: classic, fat poor, tuberous sclerosis associated and epithelioid. J Urol. 2008; 180:836-43.

32. $\mathrm{Xu} Z \mathrm{ZF}, \mathrm{Xu} \mathrm{HX}, \mathrm{Xie} \mathrm{XY}$, et al. Renal cell carcinoma and renal angiomyolipoma: differential diagnosis with real-time contrast-enhanced ultrasonography. J Ultrasound Med. 2010;29:709-17.

33. Lu Q, Wang W, Huang B, et al. Minimal Fat renal Angiomyolipoma: the initial study with contrast-enhanced ultrasonography. Ultrasound Med Biol. 2012;38:1896-901.

34. Siegel R, Naishadham D, Jemal A. Cancer statics. CA cancer J Clin. 2012;62:10-29.

35. Zhang $\mathrm{C}, \mathrm{Li} \mathrm{X}, \mathrm{Hao} \mathrm{H}$, et al. The correlation between size of renal cell carcinoma and its histopathological characteristics: a single center study of 1867 renal cell carcinoma cases. BJU Int. 2012;110(11 Pt. B):E481-5.

36. Jiang J, Chen Y, Zhou Y, et al. Clear cell renal cell carcinoma: contrast-enhanced ultrasound features relation to tumor size. Eur J Radiol. 2010;73:162-7.

37. XuZF, Xu HX, Xie XY, et al. Renal cell carcinoma: real-time contrastenhanced ultrasound findings. Abdom Imaging. 2010;35:750-6.

38. Gerst S, Hann LE, Li D, et al. Evaluation of renal masses with contrast-enhanced ultrasound: initial experience. AJR Am J Roentgenol. 2011;197:897-906.

39. Ignee A, Straub B, Brix D, et al. The value of contrast enhanced ultrasound (CEUS) in the characterization of patients with renal masses. Clin Hemorheol Microcirc. 2010;46:275-90.

40. Catalano O, Lobianco R, Sandomenico F, et al. Splenic trama: evaluation with contrast-specific sonography and a second generation contrast-medium. J Ultrasound Med. 2003;22:466-77.

41. Valentino M, Serra C, Zironi G, et al. Blunt abdominal trauma: emergency contrast-enhanced sonography for detection of solid organ injuries. AJR Am J Roentgenol. 2006;186:1361-7.

42. Regine G, Atzori M, Miele V, et al. Second-generation sonographic contrast agents in the evaluation of renal trauma. Radiol Med. 2007;112:581-7.

43. Fischer T, Dieckhofer J, Muhler M, et al. The use of contrast-enhanced US in renal transplant: first results and potential clinical benefits. Eur Radiol. 2005;15(Suppl. 5):E109-16.

44. Grzelak P, Szymczyk K, Strzelczyk J, et al. Pefusion kidney graft pyramids and cortex in contrast-enhanced ultrasonography in the determination of the cause of delayed graft function. Ann Transplant. 2011;23:48-53.

45. Kihm LP, Hinkel UP, Michael K, et al. Contrast enhanced sonography shows superior microvascular renal allograft perfusion in patient switched from cyclosporine A to everolimus. Transplantation. 2009;88:261-5.

46. Kay DH, Mazonakis M, Geddes C, et al. Ultrasonic microbubbles contrast agents and the transplant kidney. Clin Radiol. 2009;64:1081-7.

47. Lebkowska U, Janica J, Lebkowski W, et al. Renal parenchima perfusion spectrum and resistive index (RI) in ultrasound examination with contrast medieum in the early period after kidney transplantation. Transplant Proc. 2009;41:3024-7.

48. Schwenger V, Korosoglou G, Hinkel UP, et al. Real-time contrast-enhanced sonography of renal transplant recipients predicts chronic allograft nephropathy. Am J Transplant. 2006;6:609-15.

49. Wilson SR, Greenbaum LD, Goldberg BB. Contrast-enhanced ultrasound: what is the evidende and what are the obstacles? AJR Am J Roentgenol. 2009;193:55-60.

50. Mouraviev V, Joniau S, Van PH, et al. Current status of minimally invasive ablative techniques in the treatment of small renal tumors. Eur Urol. 2007;51:328-36. 